\title{
Start Date
}

National Cancer Institute

\section{Source}

National Cancer Institute. Start Date. NCI Thesaurus. Code C68616.

The calendar date on which something is to start or did start. 